\title{
Customer-focused science for environmental sustainability: a continuing case study from the NSW Government
}

\author{
Summerell, G., Leys, J., Booth, S., Oliver, I., Wilson, K., Littleboy, M. and Jenkins, B. \\ NSW Office of Environment and Heritage, PO Box 5336 Wagga Wagga NSW 2650 Email: \\ gregory.summerell@environment.nsw.gov.au
}

\begin{abstract}
This paper presents the progress of a new scientific branch in the New South Wales Government that aims to maximise customer service and optimise the management and sharing of knowledge. Summerell et al. (2013) presented the reasoning and design concepts used to establish the Ecosystem Management Science Branch (EMS) in the Science Division of the NSW Office of Environment and Heritage (OEH). EMS consists of new staff positions placed in cross-functional teams, in preference to discipline-based teams. Six teams were designed to collaboratively deliver research services and products to customers and colleagues who undertake environmental management, and develop and implement policy and programs. Two core objectives were adopted to underpin and progressively transform the work of EMS Branch. These were to:
\end{abstract}

(1) enhance knowledge management and customer engagement as a means of empowering community and government decision-making, and better achieving environmental sustainability;

(2) capitalise on our science leaders, and further develop them using the concept of 'scientist' social capital, which we define as benefits that arise from the science practitioner's reputation, status and relationships.

Traditionally, environmental science has struggled to embrace the social dimensions and implications of people in the landscape. This has reduced the incorporation of science into policy and regulation, and its use at all levels of decision making. This paper reports on the progress of these two core objectives over the last two years.

The EMS Branch has the challenge of integrating a wide range of highly diverse research areas to address issues of importance to the NSW Government. Six science leaders and one Director lead 65 staff who are located throughout NSW and undertake 134 projects throughout Australia. An early initiative of EMS Branch was to identify and map staff against projects. This improved transparency and accountability on how resources were being allocated. To provide strategic direction to EMS, the Director and Senior Team Leaders (STLs) developed a Program Logic to help consolidate, manage and communicate the purpose of our work.

Developing the EMS Branch Program Logic provided a shared clarity in direction and purpose of current activities and staff roles consistently with a long-term vision. It also demonstrated how EMS Branch research contributes to the goals of Science Division, OEH and the NSW Government. A bonus of the Branch Logic is that it enabled better communication and more capacity to influence and engage our customers and the organisation.

A case study is presented to demonstrate early benefits arising from pursuing the Branch's two core objectives. The project termed the Grazing Study seeks to provide comprehensive knowledge to inform evidence-based decision-making by the NSW Government for social, economic and ecological impacts for the continuation of livestock grazing in certain areas of parks and reserves managed by the New South Wales National Parks and Wildlife Service.

Examination of initial project results and outcomes suggest that the impact of our science is increased by using 'scientist' social capital, combined with a customer focus and collaboration with a range of partners. This approach produced an information exchange based on relationships and trust. A productive private-public sector partnership has increased the project's reach and delivery through greater awareness and shared understanding. Ultimately we expect our model, based on our two core objectives, to result in further impact of our science.

Keywords: Environmental science impact, science sustainability, government science, knowledge management, matrix management 
Summerell et al., Customer-focused science for environmental sustainability: a continuing case study from the NSW Government.

\section{INTRODUCTION}

Innovative reasoning and design were used to establish the Ecosystem Management Science Branch (EMS) within the Science Division of the NSW Office of Environment and Heritage (OEH) (Summerell et al. 2013). The nature of EMS and its work lends itself to matrix (cross-functional) management. To enable greater linkages, and for matrix managed projects to be more collaboratively developed and implemented, six teams headed by science leaders were created. The process of defining the teams was iterative and involved direct consultation with all staff members. This resulted in the teams being based on functions (i.e. Assessment, Processes, Evaluation, Conservation, Restoration and Knowledge Services) and not a science discipline. This enables easier matrix flow of tasks (Bella and Williamson 1986; Rodgers 2006, and Cullen, 1990).

The EMS Branch was formed in response to NSW 2021 (NSW Government, 2011) which is the 10 year strategic plan for the NSW Government that guides its business and decision-making. One of the five goals of NSW 2021 focuses on improving people's lives by protecting natural environments and building a strong sense of community. There are considerable challenges for EMS to help the government and diverse communities move towards environmental sustainability. Biophysical interactions are not easily understood due to the inherent levels of complexity. Further to this, people are an intrinsic part of our ecosystems and there is need to understand the cultural, social, and economic interactions before advice is offered about how to best sustain Ecosystems. The differing values of communities concerning our environment and natural resources, ultimately leads to trade-offs for the resource utilisation. Government acts on these trade-offs with the intent of making socially-responsible decisions. Summerell et al. (2013) describe how science needs to play a greater role in this decision-making by providing knowledge and transparency that better informs and supports such decisions. Von Winterfeldt (2013) presents a framework for bridging the gap between science and decision- making. He explains how a transparent and formal analysis of beliefs and values, using skills from both areas, can be a helpful process to facilitate improvements.

The EMS Branch is interdisciplinary with 65 staff in 15 locations across NSW. Our staff address a wide range of issues of importance to the NSW Government, including: landscape health (soils and vegetation); land use and planning; threatened and native species conservation; ecological management of pest, weeds and invasive species; restoring biodiversity and ecological processes; connecting country, cultures and science; fire ecology and risk management; and assessing impacts and enhancing capacities to adapt to climate change.

We have established two core objectives in the Branch which underpin our work, namely:

(1) To enhance knowledge management and customer engagement as a dual means of empowering community and government decision-making towards improved environmental sustainability;

(2) To capitalise on, and further develop, our science leaders using the concept of 'scientist' social capital; which we define as, benefits that arise from the science practitioner's connections, reputation, status and relationships.

Our construct and use of the term 'scientist' social capital, is consistent with original and subsequent definitions of social capital. Bordieu (1986) first defined this as the aggregate of the actual or potential resources which are linked to possession of a durable network of more or less institutionalized relationships of mutual acquaintance and recognition. Coleman (1990) and Putnam (1993) subsequently promoted social capital as the web of cooperative relationships between citizens (in our case, scientists) that facilitates resolution of collection action problems. Since then, the OECD has used a broader definition, to include shared norms, values and understandings that facilitate co-operation within or among groups (Keeley 2000).

Traditionally environmental science has struggled to consider and embrace the social dimensions and implications of people in the environment, so science is often not adopted (Ryder D et. al. 2010, Richard, et. al. 2015). This diminishes the outcomes from environmental research. To unlock our ability in understanding social dimensions of a project we also need to consider the scientist's social capital. We believe that such capital enables greater capacity and responsiveness to deliver science across diverse subjects, by creating more opportunities for resourcing as well as and developing wider professional and social engagement. 
Summerell et al., Customer-focused science for environmental sustainability: a continuing case study from the NSW Government.

\section{UNDERSTANDING OUR WORK, WHY WE DO IT AND HOW WE TELL OTHERS}

In 2013, the EMS Branch finished recruiting its permanent staff positions. Nearly all of these (95\%) were "new" positions designed to meet the new objectives of government and filled through competitive, merit-based selection processes. Staff were experts in their fields and many had existing projects. To honour core objective 1, the science leaders agreed to fulfil existing contracts with our long term customers.

In addition to NSW 2021, another driver of our research directions is the OEH Knowledge Strategy (http://www.environment.nsw.gov.au/knowledgestrategy/). It has six knowledge themes and each knowledge theme has a goal to help address priority knowledge needs. To achieve the goals of mainly the Landscape Management and Biodiversity Themes, EMS science leaders and staff worked alongside colleagues from across OEH (organisation size of $\sim 4000$ staff) to develop, high priority, integrated and multi-disciplinary OEH-wide projects. This process placed a strong focus on collaborating with our internal clients in $\mathrm{OEH}$, and allowed teams to design and subsequently implement projects from initiation, consistent with core objectives 1 and 2 .

Once all existing and new projects were identified, the EMS Branch mapped the Full Time Equivalent (FTE) commitments of staff to 134 projects. For the first time, the Branch had transparency and clearer accountability for projects. Given the regional location of many staff, this product became an important communication tool enabling them to identify each other's project across the Branch. However, because of the variety of projects being undertaken, the Branch's work now appeared diffused and not strategic.

To overcome this issue, the science leaders are developing a Program Logic to manage and communicate the Branch's research and work program. The following vision, mission and goals will also be pursued consistent with core objectives 1 and 2 .

Vision: Ecosystems, plants and animals are better conserved through improved landscape management.

Mission: We lead and undertake rigorous environmental science and provide advice informed by cultural and community values.

Goals: People are better able to understand and appreciate the environment in their decision-making.

Cultures are strengthened and shared.

Better management responses to climate change.

Landscapes are better managed.

Plants and animals are better conserved.

Program Logic helps to get your thinking right. It is a useful planning, communication and evaluation tool as it articulates what the program is, what it expects to do, and how success will be measured. It is useful for checking the proposed program design for adequacy of cause and effect, and the reasons or assumptions behind this (www.ghd.com/pdf/4.\%20Program\%20Logic.pdf). The draft EMS Branch Logic is already providing clarity of direction and long term vision. It has set the strategic direction for our work, clarifying purpose and demonstrating how projects contribute to the goals and programs of OEH and the NSW Government.

The draft EMS Branch Logic has also facilitated a more fruitful dialogue with our customers. The placement of projects into functional groups (termed Sub-Programs) that relate directly to corporate and government goals provides a structure that more clearly conveys to our customers the strategic thrust of our many and varied projects. Projects are progressively being focused on the Sub-Program intermediate outcomes they can achieve and the ultimate outcomes to which they can contribute. This is a significant step forward from the previous disciplinebased outputs (for example, scientific papers and other products and services).

The Outcomes Hierarchy of the draft Branch Logic comprehensively embraces the Branch's two core objectives. Figure 1 highlights the extent to which the outcomes are underpinned or integrate knowledge management and customer engagement (1) and scientist social capital (2). Of the 17 outcomes, 11 relate to knowledge management and customer service, four to scientist social capital and three to both core objectives 1 and 2 . 
Summerell et al., Customer-focused science for environmental sustainability: a continuing case study from the NSW Government.

\section{Immediate Outcomes}

1. Our work is of high priority, clearly articulated and with a stated rationale.

2. Staff are valued and supported to enhance their knowledge and capabilities. (1) and (2)

3. The breadth of our skills and depth of knowledge are maintained and enhanced. (1) and (2)

4. Context and social dynamics inform activity design and the interpretation of results. (1)

5. Our staff and colleagues understand and support what we do and why we do it. (1)

6. EMS is dynamic, responsive and adaptable in foreseeing and meeting emerging issues. (2)

7. Research and advice is in accordance with our mission and customer needs. (1)

8. Science-based evidence informs policy and shape management options for the environment. (1) and (2)

\section{Intermediate Outcomes}

9. Better understanding of interactions within biodiversity, landscapes and cultural systems and their processes and threats (1)

10. Community and cultural values and perspectives on biodiversity and landscape management are respected.

11. Improved effectiveness of policy, planning, and decision-making at all levels. (1) and (2)

12. Improved monitoring and assessment of changes in biodiversity, landscapes and culture. (1)

13. Our partners and customers benefit from the science and the knowledge we create together. (1)

14. Improved ecological understanding underpins adaptation to climate change. (1)

15. Our collaborative science delivers rigorous, practical and relevant solutions that people use. (1)

16. Our leadership and capabilities in ecosystem science delivers conservation, cultural and ecosystem service benefits. (2)

17. Our science identifies emerging knowledge needs and informs Science Division and OEH priorities. (1)

Figure 1. Outcomes Hierarchy for Draft EMS Branch Logic highlighting the outcomes which integrate or are underpinned by branch core objectives (1) and (2).

\section{Case Study: The NSW Grazing Study; Ecological Science Component}

Following a request by the NSW Government, the OEH established a cross-departmental project to investigate the impacts and benefits of livestock grazing in selected River Red Gum, Black Box, and Cypress Pine parks and reserves, managed by the NSW National Parks and Wildlife Service (NPWS). The project (hereafter 'the Grazing Study') aims to examine the social, economic and ecological impacts and benefits of grazing in certain NPWSmanaged areas in southwest NSW. It seeks to provide comprehensive knowledge to inform evidence-based decision-making by the NSW Government about the continuation of grazing in certain areas of national parks where grazing permits exist.

The EMS Branch has the major responsibility for the design and delivery of the ecological science components of the Grazing Study. High level expertise has been required in quality control of the study design and implementation. A large component of the project (not reported here) relates to stakeholder consultation, economic analysis, and other issues which are generally beyond the scope of the science undertaken by EMS Branch. Consistent with our core objectives, our customer, scientist social capital and outcomes orientation demands that our delivery and information flow considers and appropriately addresses any relevant arising needs. 
The EMS Branch has led the development of the scientific methodology that underpins the Grazing Study. Customer focus, knowledge management and the social capital of the EMS Branch scientists have each been important and assisted the project in the following ways:

Achievements: A rigorous study design was co-developed with our "partners": the (NPWS) and the study's independent facilitator. It was reviewed by an independent external panel of experts. Implementation was undertaken by EMS and NPWS staff in collaboration with private contractors and the Tertiary sector, and oversighted by the independent facilitator. Data analysis drew heavily upon the international and national intellectual credibility of EMS scientists and their networks. This increased both the profile and rigour of the project to the extent that we expect wider acceptance of this large scale study on completion.

Figure 2 is a representation of where all science projects undertaken by EMS and the other six branches in the $\mathrm{OEH}$ Science Division interact with various groups and partners. It is argued that the OEH science project delivery model engages all relevant groups and thus achieves a wider science delivery and impact. Goggin et al, (2015a,b) provides evidence of the breadth and depth of our reach through the mapping of connections in delivering our work. The example of the Grazing Study outlines how the EMS Branch scientist's social capital networks, in most cases, provided efficient and effective access to the pertinent components in Figure 1.

\section{Grazing study}

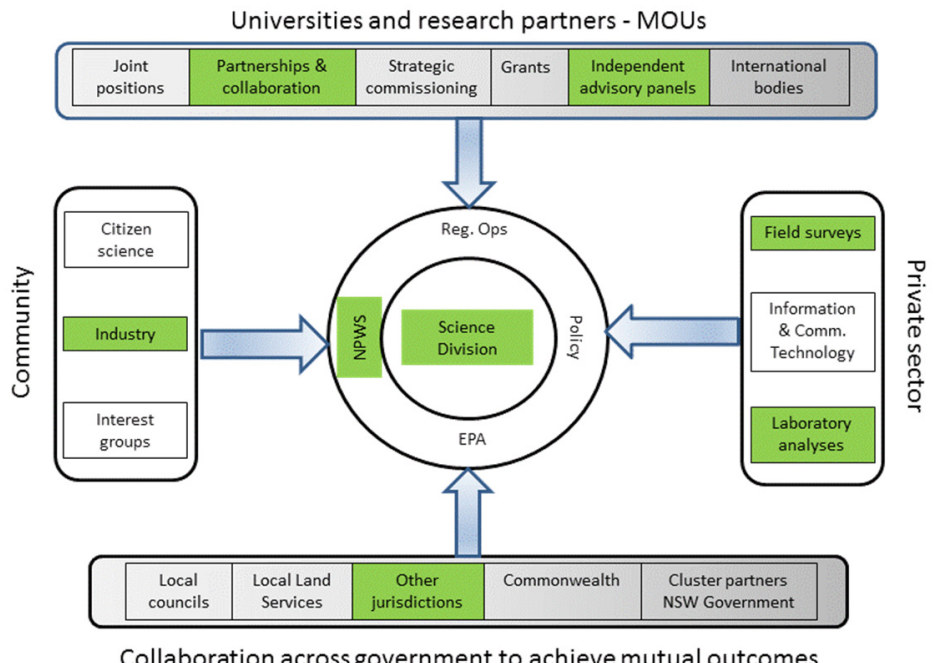

Figure 2. Case study showing the science connections for project (NSW Grazing Study) delivery (described above). The Science Division is positioned at the centre of a network to provide the majority of science to OEH. The role of the Science Division is both to deliver science directly to NPWS, Regional Operations (Reg. Ops),

Policy and the Environmental Protection Agency (EPA); and to facilitate, collaborate, commission, source, influence and/or directly contract science from partner organisations.

Partnerships and Collaborations: The University of NSW and University of New England are key partners in the Grazing Study. Fifteen precent of EMS Branch scientists are strategically located on university campuses to facilitate these partnerships. This enables more efficient and effective access to additional resources (for example, discipline-specific academics, student researchers and specialist equipment).

Independent Advisory Panels: an independent facilitator who reports to the responsible NSW Minister and chairs the internal steering committee for the Grazing Study project oversees the Study. The facilitator oversights all assessments of the social, economic and ecological impacts and benefits of grazing. The role also provides the interface between stakeholders, the project team and the Minister, and supplies regular updates to interested parties.

In 2013, the independent River Red Gum (RRG) scientific advisory panel was engaged to guide the Study's survey design. In October 2014 a second independent expert panel was convened to provide critique and feedback on the proposed analysis of the ecological and biodiversity data generated by the study. The outcomes from this committee were endorsed by the RRG panel. 

NSW Government.

Industry: Stakeholder workshops were held to provide updates on the Study but more importantly to seek advice and input the stakeholder's views for important ecological and biodiversity components that should be considered for analyses. These workshops engaged stakeholders during implementation and increased mutual understanding of what was considered important by scientists and stakeholders alike. At the time of writing, workshop reports are being finalised for distribution to stakeholders.

Field Surveys: The field work for the Grazing Study was undertaken in 2013 and 2014 across various land tenures. Vegetation, grazing pressure and soil health was surveyed across 451 sites from the Riverina to Central West Region of NSW. Approximately $65 \%$ of the field budget (the main component of the project) was outsourced to the private sector to complement $\mathrm{OEH}$ capacity to deliver the project. This also increased the breadth of people engaged in delivering the project. The independent facilitator oversight in combination with OEH Science and NPWS collaboration, maintained quality control of all ecological science components. In 2014/15 a comprehensive fauna component of the study was undertaken using the Study's established principles and processes.

Over 182,000 vegetation and soil data records were collected by the Grazing Study during 2013/14. The EMS branch functional teams created the impetus to identify and budget for such knowledge management issues in accordance with the Branch Logic. As a result these biodiversity records were corporately managed and are to be made publicly accessible on completion of the Grazing Study. This will provide data transparency, safe storage of data and increase the opportunity for value-adding to the science investment, as other researchers and stakeholders access the data.

Laboratory Analysis: Rapid, high quality scientific analysis of the Grazing Study's soil samples was required. The partnerships and connections of EMS Branch scientists enabled the Grazing Study to rapidly engage laboratories with new technology. The technology enabled 1950 soil samples to be analysed for particle size composition and chemistry and 750 samples to be processed for additional chemicals.

Other Jurisdictions: The issue of grazing in national parks has received considerable attention in Victoria. The findings of the Grazing Study in NSW maybe relevant to other jurisdictions. Fire management/incident organisations also have a strong interest in relation to the potential impact of grazing in reducing fuel loads. Due to this interest, the Grazing Study's field data collection included the fire authorities' assessments methods for overall fuel hazard.

NPWS: Staff from NPWS were intimately involved in the project design and implementation. As the land managers, NPWS have provided funding, practical direction and solutions to the survey design and implementation. This is in addition to providing field support, project governance and overall management of the Grazing Study.

\section{CONCLUSIONS}

Early experience with the innovative EMS Branch delivery model and applying our two core objectives for knowledge management, customer focus and scientists' social capital, suggests the program logic delivery model and EMS core objectives can work together to enhance the processes used to inform government decision-making for environmental sustainability. The Grazing Study provides initial evidence of how the reach and impact of EMS Branch science has practically improved as a result.

EMS Branch core objective 1 is to enhance knowledge management and customer engagement as a dual means of empowering community and government decision-making towards improved environmental sustainability. The core objective acknowledges that people are the pivotal element of our science delivery. The case study highlights the importance of involving collaborators in developing and delivering projects. Although this can be challenging for scientists (due largely to discipline-based cultural differences) (Goggin ${ }^{\mathrm{B}}$ et. al. 2015), it is essential to maximise customer satisfaction and use of knowledge and information generated from scientific projects (Goggin ${ }^{B}$ et. al. 2015). It is a core commitment for staff in EMS Branch, and expressed in our draft Branch Logic.

EMS Branch core objective 2 is to capitalise on, and further develop, all science leaders, using the concept of scientist social capital. As evidenced by the case study, this core objective fosters and enables a focused, responsive and ready capacity for project development and delivery relating to management of environmental issues. This is especially important for government scientists who must respond to changes in government priority, and to local and regional implications of emerging complex and global issues pivotal to the EMS Branch success, 'scientist' social capital underpins the draft Branch Logic. 
Summerell et al., Customer-focused science for environmental sustainability: a continuing case study from the NSW Government.

To demonstrate the benefit of the connections of our scientists we have documented in a separate study the social networks of EMS scientists to identify who they are working with. This will help identify gaps in connections to key customers $\left(\right.$ Goggin $^{\mathrm{A}}$ et. al. 2015). We have also explored the factors that customers describe as the most important when developing working relationships with our scientists. Our intention is to foster these factors in EMS Branch so we undertake work which has the best chance of leaving a lasting legacy for both the customer and the environment $\left(\operatorname{Goggin}^{B}\right.$ et. al. 2015).

\section{ACKNOWLEDGEMENTS}

The proofing and advice provided to prepare this paper by Dr. Louise Goggin, Dr. Nicholas Carlile and Mr Mark Peacock are gratefully acknowledged.

\section{REFERENCES}

Bella, D.A and Williamson, K.J (1986). Conflicts in interdisciplinary research. Interdisciplinary Analysis and research, 6, 347-354.

Coleman, J. S. (1990). "Foundations of Social Theory”. Cambridge: Belknap Press of Harvard Univ. Press.

Cullen, P. (1990). The turbulent boundary between water science and water management. Freshwater Biology, 24, 201-209.

Goggin, C.L., Grieve, A., Waters, S., Summerell, G. K., Oliver, I., Leys, J., Barrett, T., Drielsma, M., McInnesClarke, S., Littleboy, M., Jenkins, B. (2015a). Working with customers: how scientists can develop successful partnerships that produce an enduring legacy. This Conference

Goggin, C.L., Cunningham, R., Summerell, G., Leys, J., Barrett, T., Auld, T., Oliver, I., and Littleboy, M. (2015b). Exploring the networks of government scientists using Social Network Analysis: a scoping study. This Conference

Keeley, B. (2007). OECD Insights: Human Capital How what you know shapes your life. Organisation of Economic Co-operation and Development (OECD).

NSW Government. (2011). NSW 2021. NSW Department of Premier and Cabinet. http://www.2021.nsw.gov.au/sites/default/files/NSW2021_WEB\%20VERSION.pdf

Putnam, R.D. (1993). The Prosperous Community: Social Capital and Public Life. The American Prospect [Internet] (13):35-42.

Richard, V., Michael, J., and McCarthy, G. (2015). Knowledge Management Research \& Practice 13(2), $187-$ 197. doi:10.1057/kmrp.2013.41; published online 19 August 2013

Rodgers, K. (2006). The real river management challenge: integrating scientists. River Research and Applications $22,269-280$.

Ryder, D.S., Tomlinson, M., Gawne, B., and G. Likens. (2010). Defining and using 'best available science': a policy conundrum for the management of aquatic ecosystems. Marine and Freshwater Research,61, 821- 828.

Summerell G., Leys, J and Wilson K. (2013). Customer Focused Science and knowledge management for sustainability in New South Wales, Australia. 20th International congress on Modelling and Simulation, Adelaide, Australia, 1-6 December 2013. Www.mssanz.org.au/modsim2013. http://www.mssanz.org.au/modsim2013/K3/summerell.pdf

Von Winterfeldt, D. (2013). Bridging the gap between science and decision making. Proceedings of the National Academy of Sciences. 110: 14055-14061. 